\title{
Ultrafast laser-assisted spatially targeted optoporation into cortical axons and retinal cells in the eye
}

Subrata Batabyal

Young-Tae Kim

Samarendra Mohanty 


\section{Ultrafast laser-assisted spatially targeted optoporation into cortical axons and retinal cells in the eye}

\author{
Subrata Batabyal, ${ }^{\text {a }}$ Young-Tae Kim, ${ }^{\text {b }}$ and \\ Samarendra Mohanty ${ }^{a, \star}$ \\ aNanoScope Technologies LLC, Bedford, Texas, United States \\ bUniversity of Texas at Arlington, Department of Bioengineering, \\ Texas, United States
}

\begin{abstract}
Visualization and assessment of the cellular structure and function require localized delivery of the molecules into specific cells in restricted spatial regions of the tissue and may necessitate subcellular delivery and localization. Earlier, we have shown ultrafast near-infrared laser beam-assisted optoporation of actin-staining molecules into cortical neurons with single-cell resolution and high efficiency. However, diffusion of optoporated molecules in soma degrades toward the growth cone, leading to difficulties in visualization of the actin network in the growth cone in cases of long axons. Here, we demonstrate optoporation of impermeable molecules to functional cortical neurons by precise laser subaxotomy near the growth cone, leading to visualization of the actin network in the growth cone. Further, we demonstrate patterned delivery of impermeable molecules into targeted retinal cells in the rat eye. The development of optoporation as a minimally invasive approach to reliably deliver exogenous molecules into targeted axons and soma of retinal neurons in vivo will enable enhanced visualization of the structure and function of the retina. () 2017 Society of Photo-Optical Instrumentation Engineers (SPIE) [DOI: 10.1117/1.JBO.22.6.060504]
\end{abstract}

Keywords: optoporation; laser microbeam; ultrafast laser; laserfection; axonal optoporation; retina.

Paper 170276LR received Apr. 27, 2017; accepted for publication Jun. 13, 2017; published online Jun. 29, 2017.

\section{Introduction}

Visualization, activation, and detection of the neuronal structure and function require targeted delivery of proteins, such as antibodies, genes encoding reporter proteins, ion channels, and voltage indicators. The introduction of foreign DNA, ${ }^{1}$ shortinterfering RNA, ${ }^{2}$ small molecules, proteins, ${ }^{3}$ and drugs into living cells, organs, and whole organisms is essential for a variety of applications in genetics, cell and developmental biology, vaccination, ${ }^{4}$ gene therapy, ${ }^{5,6}$ and other therapeutic strategies. Further, the transfection of plasmids encoding fluorescent proteins ${ }^{7}$ is routinely used to visualize cellular and subcellular

*Address all correspondence to: Samarendra Mohanty, E-mail: smohanty@ nanoscopetech.com structures and also to study various functional aspects of cell and developmental biology. ${ }^{8}$ To visualize and control physical connection, expression pattern, and neural activity, it is necessary to deliver genes encoding fluorescent molecules (proteins for labeling), ion channels (for activation by light), genetically encoded voltage indicators (for detecting neural activity), and other molecules, such as antibodies/fluorescence dyes targeted to intracellular organelles. Further, for understanding of the neuronal function, it will be useful to localize the expression of the targeted molecules in neurons in restricted spatial regions and even subcellular regions. Traditional methods (e.g., lipidbased transfection or electroporation) for delivery lack the ability of spatially targeted delivery. Therefore, there is an urgent need for development of a minimally invasive approach to reliably deliver probe molecules into targeted neurons for visualizing the structures and assessing the functions. Furthermore, neurological disorders having spatial aberrations such as geographic atrophies of the retina will benefit from targeted delivery of various therapeutic drugs or small molecules in spatially targeted cells or tissue.

The membranes of targeted cells can be transiently perforated in a minimally invasive manner by use of a tightly focused pulsed laser beam, allowing exogenous molecules to enter the cell. Over the last decades, laser-assisted perforation has been applied to inject macromolecular substances into single plant and animal cells in a noncontact and noninvasive manner. There is considerable interest in optically transfecting (optoporation) cells using ultrafast-pulsed light ${ }^{9-18}$ because of its selective targeting capability, higher efficiency, and viability ${ }^{9,17}$ as compared to other methods. The femtosecond (fs) near-infrared (NIR) laser-based transfection has been shown to be safe, providing high efficiency and survival of optoporated embryo during development, ${ }^{19}$ as well as for in-vivo gene delivery. ${ }^{20}$ Earlier, we demonstrated the use of ultrafast NIR laser microbeam for spatially localized transfection of opsin-encoding genes into neurons in retinal explant ${ }^{10}$ as well as into other mammalian cells. ${ }^{21}$ Though ultrafast optoporation-based delivery provides an increased efficiency of gene transfer in cells and several tissues, ${ }^{9,10,20}$ optoporation at axonal level has not been demonstrated so far. Though optoporated molecules (in soma) can diffuse to the growth cone, the diffusion becomes weaker when the growth cone is far from soma. Here, we report that impermeable actin-staining dyes can be reliably delivered into axons of living cortical neurons, allowing rapid localization in the growth cone. Further, we demonstrate the use of ultrafast laser microbeam for targeted delivery of molecules to the retina in intact rat eye.

\section{Methods}

All experimental procedures were conducted according to the Institutional Animal Care and Use Committee approved protocol.

\subsection{Neuron Isolation and Culture}

The cortical neurons were isolated from embryonic 18-day rat embryos. The cortical tissues were dissected, cleaned (meningeal layer), and enzymatically dissociated $(0.125 \%$ trypsin in $\mathrm{L}-15$ medium) for $20 \mathrm{~min}$ at $37^{\circ} \mathrm{C}$. The dissociated cortical neurons $(100,000 /$ device $)$ were seeded on poly-D-lysine $(0.01 \%$,

$1083-3668 / 2017 / \$ 25.00$ @) 2017 SPIE 
Sigma) precoated coverglass with polydimethylsiloxane barrier (Sylgard 184, Dow Corning), and the serum-free culture medium (neurobasal medium supplemented B-27 with brain derived neurotrophic factor and NT-3, $10 \mathrm{ng} / \mathrm{ml}$ ) was changed every 3 days.

\subsection{Eye Isolation and Intravitreal Injection}

Whole eyes were isolated from the adult rat by dissection and immediately used for optoporation. The connective muscles from the eye were cleared, and the eye was kept inside a well filled with HEPES buffer solution. $5 \mu \mathrm{l}$ of Alexa 594 phalloidin solution $(1 \mu \mathrm{M})$ was injected to the intravitreal space using a Hamilton microsyringe inserted through the sclera into the vitreous cavity. This amounts to final concentration of $100 \mathrm{nM}$ in the vitreous, considering the vitreous volume of $\sim 50 \mu \mathrm{l}$. Within $15 \mathrm{~min}$, optoporation of the retina was carried out using the ultrafast NIR laser beam. The cornea was kept moist with a balanced salt solution during the entire surgical procedure.

\subsection{Optoporation Setup}

A Ti:sapphire laser (Newport Spectra-Physics, Inc.) beam tuned at $790 \mathrm{~nm}$ with repetition rate: $80 \mathrm{MHz}$ and pulse width of $\sim 200$ fs was coupled to an inverted fluorescence optical microscope (Nikon) by a dichroic mirror. A $100 \times(\mathrm{NA}=1.3$ and working distance: $0.2 \mathrm{~mm}$ ) microscope objective was used to focus the laser beam to diffraction-limited spot (radius: $\sim 370 \mathrm{~nm}$ ) on the axon. For optoporation of the retina in intact rat eye, a long working distance $10 \times(\mathrm{NA}=0.25$ and working distance: $15 \mathrm{~mm}$ ) microscope objective was used. The exposure time at each optoporated site was controlled by an external electro-mechanical shutter (Uniblitz Inc.). The fluorescence excitation light (through excitation bandpass filter) from the mercury lamp was directed to the sample by a dichroic mirror, and the emitted fluorescence was selected by an emission filter in epifluorescence mode. An IR cutoff filter was used to block the backscattered laser light. Fluorescence and bright-field images were acquired before and after optoporation using a cooled EMCCD camera (Photometrics) and processed with ImageJ (NIH) software.

\section{Results}

\subsection{Optoporation of Impermeable Molecules into Growth Cone by Laser Subaxotomy}

In the case of the growth cone far from the soma, diffusion of optoporated molecules at soma will require significant time or often is not able to reach the growth cone. For labeling the growth cone with impermeable actin-binding molecule (Alexa 594 phalloidin), we optoporated axonal shaft near the growth cone. Tightly focused (by $100 \times$ microscope objective with $\mathrm{NA}=1.3$ and working distance: $0.2 \mathrm{~mm}$ ) ultrafast fs laser was used to produce subaxotomy-level injury in a highly localized manner. Figure 1(a) shows bright-field image of axons far $(\sim 1 \mathrm{~mm})$ from the soma of rat cortical neurons. The time-lapse fluorescence images of axon of cortical neuron optoporated with Alexa 594 labeled phalloidin using ultrafast laser microbeam are shown in Figs. 1(b)-1(f). The dye can be seen to diffuse from the site of optoporation (encircled) along the axon to the growth cone. The morphology of the actin-stained lamellipodium and filopodia of the growth cone is clear with time progression.
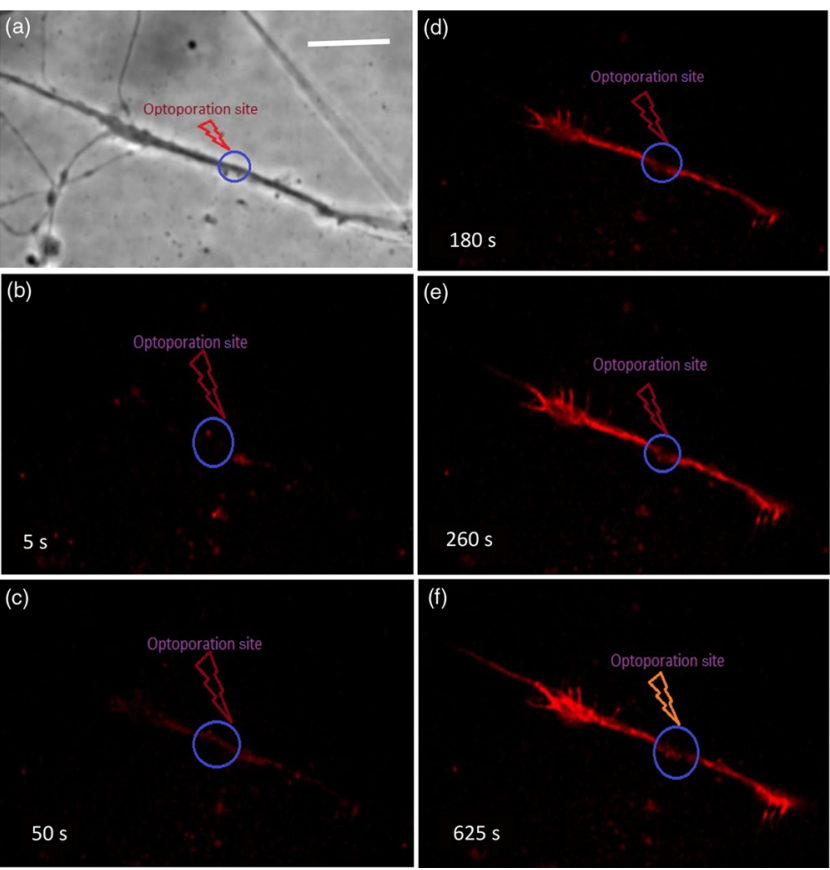

Fig. 1 Axonal optoporation: (a) bright-field image of axons $(\sim 1 \mathrm{~mm})$ far from the soma of rat cortical neurons and (b)-(f) time-lapse fluorescence images of Alexa 594 labeled phalloidin optoporated into axon of cortical neuron using ultrafast laser microbeam. Site of optoporation is encircled. Scale bar: $10 \mu \mathrm{m}$.

Since the subaxotomy damage near growth cone was found to reseal within $\sim 15 \mathrm{~min}$ of optoporation, the rise of intraaxonal fluorescence intensity due to optoporated actin-staining molecules is stabilized.

Our experiments determined that the axonal growth rate is not perturbed below laser exposure of $20 \mathrm{~ms}$ at $0.7 \mathrm{~nJ} /$ pulse after one perforation in the axon. Furthermore, while phalloidin is known to be cytotoxic, our earlier experiments have demonstrated $^{22}$ that below certain concentration $(100 \mathrm{nM})$ the optoporated neurons' viability was not compromised.

\subsection{Patterned Optoporation into Retina of Rat Eye}

Eye provides better access for NIR laser-based optoporation of the retina as compared to other organ tissues. We used a long working distance $10 \times$ microscope objective $(\mathrm{NA}=0.25$ and working distance: $0.2 \mathrm{~mm}$ ) for optoporating retinal cells in rat eye. Higher pulse energy $(1.5 \mathrm{~nJ})$ was used for these experiments. Left panel in Fig. 2 shows the schematic of optoporation of rat eye. In Figs. 2 and 3, we show spatially patterned optoporation in rat eye. Figure 2 (right panel) shows circularly patterned optoporation of Alexa-594 labeled phalloidin molecules into the retina. The time-lapse fluorescence images of progressively patterned optoporation of Alexa 594 labeled phalloidin into retinal cells of rat eye using ultrafast NIR laser beam are shown in panels (b) to (h). The bright-field image (superimposed over fluorescence) after ultrafast laser-based patterned optoporation is shown in Fig. 2(i). Figure 3 shows a triangularly patterned optoporation of the retina in rat eye in a progressive manner. Figure 4 shows another example of spatially patterned optoporation of the retina in rat eye. The optoporation sites can be seen to have residual signs (dark dots, marked by arrows) of resealed holes as shown in bright-field image in Fig. 4(f) (white arrows). 


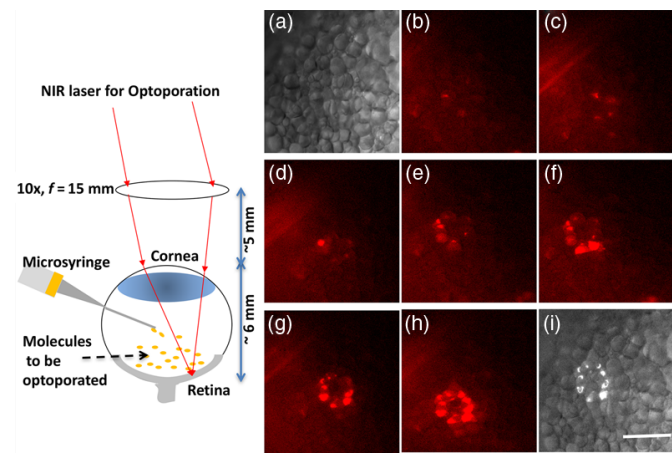

Fig. 2 Circularly patterned optoporation in rat eye. Left: schematic of optoporation of rat eye. Right: (a) bright-field image of retinal cells in a rat eye. (b)-(h) Time-lapse fluorescence images of progressively patterned optoporation of Alexa 594 labeled phalloidin into retina cells of rat eye using ultrafast NIR laser beam. (i) bright-field image (superimposed over fluorescence) after ultrafast laser delivery. Scale bar: $50 \mu \mathrm{m}$.
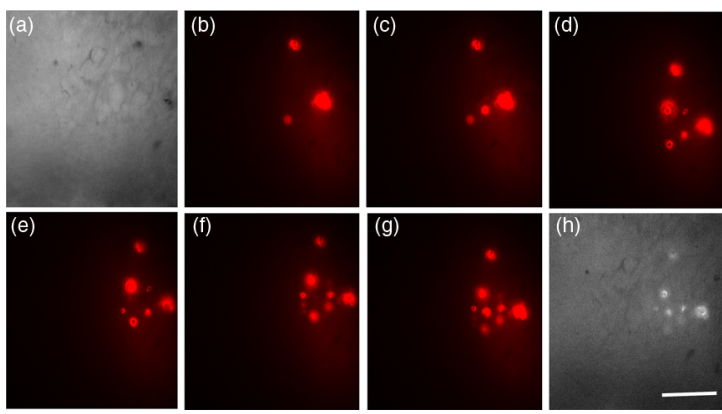

Fig. 3 Triangularly patterned optoporation the retina in rat eye. (a) Bright-field image of retinal cells in the rat eye. (b)-(g) Timesequence fluorescence images of triangularly patterned optoporation of phalloidin into the retina. (h) Bright-field image (superimposed over fluorescence) after patterned optoporation. Scale bar: $50 \mu \mathrm{m}$.
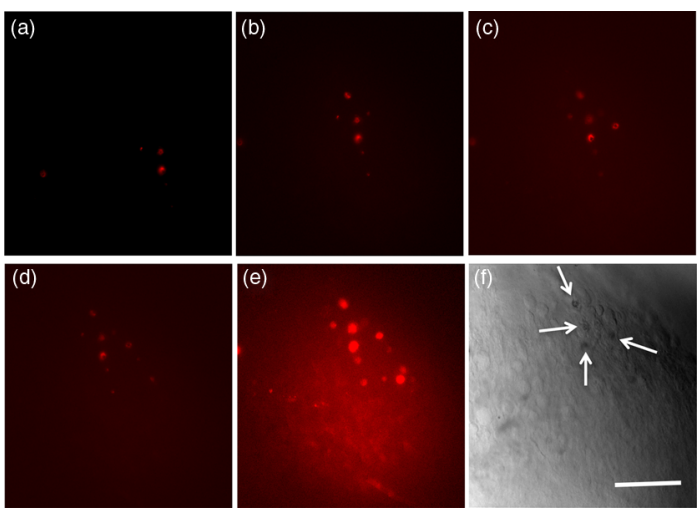

Fig. 4 Optoporation of the retina in rat eye. (a)-(e) Time-lapse fluorescence images of Alexa 594 labeled phalloidin delivered into retinal cells of rat eye using ultrafast NIR laser-assisted optoporation. (f) Bright-field image after laser delivery shows arrow-marked dark dots (resealed holes). Scale bar: $50 \mu \mathrm{m}$.

\section{Discussion}

Our results show optoporation using focused NIR ultrafast laser beams for targeted delivery of impermeable molecules into retinal cells of rat eye with single-cell resolution. Our experiments pave a way of the possibility of using fs laser as a viable tool to deliver a therapeutic gene in the retina for treatment of eye diseases in a minimally invasive manner. Further, our data showed that optoporated molecules diffuse to the growth cone upon optoporation in the axon. The ultrahigh intensity that is present in a tightly focused fs pulse creates a site-specific transient perforation. ${ }^{23}$ Precisely, localized pores can be created in axon and soma using the fs NIR laser microbeam by nonlinear interaction between ultrafast laser beam with cell. The development of optoporation as a minimally invasive approach to reliably deliver exogenous molecules into targeted axons and soma of retinal neurons in vivo will enable better visualization of the structure and function of the retina.

Furthermore, advancement of the in-vivo optical delivery methods will lead to a new approach for treating patients with retinal degeneration by first determining the degenerated areas such as geographic atrophies in dry-age-related macular degeneration followed by conventional intravitreal injection of therapeutic molecules, such as opsin-encoding genes and NIR laser-assisted, targeted nonviral delivery of the molecules to retinal cells in the degenerated areas in an efficient and minimally invasive manner.

To conclude, we have demonstrated optoporation of impermeable molecules to both axons of cortical neurons and soma of retinal cells in rat eye by precise and patterned laser microirradiation. Development of optoporation technology to reliably deliver exogenous molecules into targeted axons and soma of retinal neurons will enable enhanced visualization of the structure and improve function of the degenerated retina.

\section{Disclosures}

The authors have no relevant financial interests in the paper. S.M. is the founder of and has equity interest in NanoScope Technologies LLC, which develops several biomedical diagnosis and therapeutic products.

\section{Acknowledgments}

The authors would like to thank Lalit Chudal and Horipoda Sarkar for help in axonal optoporation experiments and Sarmishtha Satpathy for eye optoporation experiments. S.M. would like to acknowledge the supports from the National Institute of Neurological Disorders and Stroke (No. NS084311) and the National Eye Institute (No. 1R01EY025717-01A1) of the National Institutes of Health.

\section{References}

1. D. Luo and W. M. Saltzman, "Synthetic DNA delivery systems," Nat. Biotechnol. 18(1), 33-37 (2000).

2. J.-Y. Yu, S. L. DeRuiter, and D. L. Turner, "RNA interference by expression of short-interfering RNAs and hairpin RNAs in mammalian cells," Proc. Natl. Acad. Sci. U. S. A. 99(9), 6047-6052 (2002).

3. K. Burridge and J. R. Feramisco, "Microinjection and localization of a 130k protein in living fibroblasts: a relationship to actin and fibronectin," Cell 19(3), 587-595 (1980).

4. O. E. Akilov et al., "Vaccination with photodynamic therapytreated macrophages induces highly suppressive T-regulatory cells," Photodermatol. Photoimmunol. Photomed. 27(2), 97-107 (2011).

5. N. Somia and I. M. Verma, "Gene therapy: trials and tribulations," Nat. Rev. Genet. 1(2), 91-99 (2000).

6. I. M. Verma and N. Somia, "Gene therapy-promises, problems and prospects," Nature 389(6648), 239-242 (1997).

7. R. Y. Tsien, "The green fluorescent protein," Аппи. Rev. Biochem. 67(1), 509-544 (1998). 


\section{JBO Letters}

8. K. Kamimura et al., "Advances in gene delivery systems," Pharm. Med. 25(5), 293-306 (2011).

9. U. K. Tirlapur and K. Konig, "Targeted transfection by femtosecond laser," Nature 418(6895), 290-291 (2002).

10. L. Gu and S. K. Mohanty, "Targeted microinjection into cells and retina using optoporation," J. Biomed. Opt. 16(12), 128003 (2011).

11. P. Soman et al., "Femtosecond laser-assisted optoporation for drug and gene delivery into single mammalian cells," J. Biomed. Nanotechnol. 7(3), 334-341 (2011).

12. F. Stracke, I. Rieman, and K. Konig, "Optical nanoinjection of macromolecules into vital cells," J. Photochem. Photobiol. B 81(3), 136-142 (2005).

13. L. E. Barrett et al., "Region-directed phototransfection reveals the functional significance of a dendritically synthesized transcription factor," Nat. Methods 3(6), 455-460 (2006).

14. C. Peng, R. E. Palazzo, and I. Wilke, "Laser intensity dependence of femtosecond near-infrared optoinjection," Phys. Rev. E 75(4), 041903 (2007).

15. X. Tsampoula et al., "Fibre based cellular transfection," Opt. Express 16(21), 17007-17013 (2008).

16. M. Lei et al., "Femtosecond laser-assisted microinjection into living neurons," J. Neurosci. Methods 174(2), 215-218 (2008).
17. A. Uchugonova et al., "Targeted transfection of stem cells with sub-20 femtosecond laser pulses," Opt. Express. 16(13), 9357-9364 (2008).

18. A. Yamaguchi et al., "Nanoparticle injection to single animal cells using femtosecond laser-induced impulsive force," Appl. Phys. A 93(1), 39-43 (2008).

19. V. Kohli and A. Y. Elezzabi, "Laser surgery of zebrafish (Danio rerio) embryos using femtosecond laser pulses: optimal parameters for exogenous material delivery, and the laser's effect on short- and long-term development," BMC Biotechnol. 8, 7 (2008).

20. E. Zeira et al., "Femtosecond infrared laser-an efficient and safe in vivo gene delivery system for prolonged expression," Mol. Ther. 8(2), 342-350 (2003).

21. K. Dhakal et al., "Optical delivery of multiple opsin-encoding genes leads to targeted expression and white-light activation," Light Sci. Appl. 4(11), e352 (2015).

22. K. Dhakal, B. Black, and S. Mohanty, "Introduction of impermeable actin-staining molecules to mammalian cells by optoporation," Sci. Rep. 4, 6553 (2014).

23. S. K. Mohanty, M. Sharma, and P. K. Gupta, "Laser-assisted microinjection into targeted animal cells," Biotechnol. Lett. 25(11), 895-899 (2003). 